\title{
Associação entre Hipovitaminose D e Síndrome da Imunodeficiência Adquirida
}

\section{Association between Hypovitaminosis D and Acquired Immunodeficiency Syndrome} Asociación entre la Hipovitaminosis D y el Síndrome de Inmunodeficiencia Adquirida

Recebido: 27/01/2020 | Revisado: 07/02/2020 | Aceito: 11/03/2020 | Publicado: 20/03/2020

\section{Beatriz Bianca Miranda Silva}

ORCID: https://orcid.org/0000-0001-7182-6377

Universidade Federal do Piauí, UFPI, Brasil

E-mail: bia.miranda.2303@gmail.com Érica Letícia Carvalho de Oliveira

ORCID: https://orcid.org/0000-0001-5873-6043

Universidade Federal do Piauí, UFPI, Brasil

E-mail: erica.carrvalho@gmail.com

Thaís Rodrigues Nogueira

ORCID: https://orcid.org/0000-0002-2401-033X

Universidade Federal do Piauí, UFPI, Brasil

E-mail: thaisnogueiranutri@gmail.com

Betânia de Jesus e Silva de Almendra Freitas

ORCID: https://orcid.org/0000-0002-7797-735X

Universidade Federal do Piauí, UFPI, Brasil

E-mail: betaniafreitas2004@yahoo.com.br

\section{Resumo}

Objetivo: Investigar na literatura o conhecimento atual sobre a associação entre a deficiência de vitamina D e a Síndrome da Imunodeficiência Adquirida. Métodos: Trata-se de revisão integrativa da literatura, realizada por meio de pesquisa de artigos originais, em português, espanhol e inglês indexados nas bases de dados Pubmed, Lilacs e Scielo. Resultados: Foram recuperados 28 artigos, dos quais $28.6 \%(\mathrm{n}=8)$ foram incluídos nesta revisão para leitura do texto integral. Observou-se que dos estudos compilados, 100\% $(n=8)$ demonstraram que a população, em geral, possui alta taxa de deficiência de vitamina $\mathrm{D}$, independente de fatores como localidade, sexo, idade, presença de HIV ou uso da TARV, sendo estes dois últimos possíveis elementos para o agravamento da deficiência de vitamina $\mathrm{D}$, o que identifica uma 
relação de via dupla entre a doença e a hipovitaminose. Conclusão: Concluiu-se que existe uma forte associação entre hipovitaminose D e a síndrome da imunodeficiência adquirida (AIDS), geralmente potencializada ou condicionada a fatores de risco intrínsecos ou de estilo de vida. Além disso, verificou-se associação entre concentrações insuficientes da vitamina em portadores e a maior vulnerabilidade a doenças oportunistas, e opostamente, a redução de infecções, de doenças crônicas e da mortalidade em portadores de HIV/AIDS com concentrações ideais de vitamina D. Embora não tenha sido o objetivo do estudo, foram identificadas também correlações significativas entre a diminuição do número de células linfócitos T CD4. Apesar disso, ressalta-se a necessidade da realização de mais pesquisas que permitam maiores esclarecimentos sobre a temática proposta.

Palavras-chave: Vitamina D; Deficiência; HIV/AIDS.

\begin{abstract}
Objective: To investigate in the literature or current knowledge about an association between vitamin D deficiency and Acquired Immunodeficiency Syndrome. Methods: This is an integrative literature review, carried out by searching for original articles, in Portuguese, Spanish and English indexed in the Pubmed, Lilacs and Scielo databases. Results: 28 articles were retrieved, of which $28.6 \%(n=8)$ were included in this review for reading the full text. He observed that the studies compiled, $100 \%(n=8)$ demonstrated that the population, in general, has high levels of vitamin D, regardless of factors such as location, sex, age, presence of HIV or use of ART, the latter two being possible elements for the worsening of vitamin D, or that identify a double-track relationship between the disease and hypovitaminosis.
\end{abstract} Conclusion: It was concluded that there is a strong association between hypovitaminosis D and an acquired immunodeficiency syndrome (AIDS), usually potentiated or conditioned by intrinsic risk factors or lifestyle. In addition, there was an association between vitamin deficiency in patients and greater vulnerability to opportunistic diseases and, conversely, a reduction in infections, chronic diseases and mortality in HIV/AIDS patients with disease statistics D. This was the objective of the study., associated correlations were also identified between the decrease in the number of CD4 T lymphocyte cells. In spite of this, the need to conduct more research that allows greater clarification on a thematic proposal is emphasized.

Keywords: Vitamin D; Deficiency; HIV/AIDS. 


\section{Resumen}

Objetivo: investigar en la literatura o el conocimiento actual sobre una asociación entre la deficiencia de vitamina D y el síndrome de inmunodeficiencia adquirida. Métodos: Esta es una revisión integradora de la literatura, realizada mediante la búsqueda de artículos originales, en portugués, español e inglés indexados en las bases de datos Pubmed, Lilacs y Scielo. Resultados: Se recuperaron 28 artículos, de los cuales el 28.6\% (n=8) se incluyeron en esta revisión para leer el texto completo. Observó que los estudios recopilados, el 100\% $(\mathrm{n}=8)$ demostraron que la población, en general, tiene altos niveles de vitamina $\mathrm{D}$, independientemente de factores como la ubicación, el sexo, la edad, la presencia de VIH o el uso de ART, siendo estos dos últimos posibles elementos para el empeoramiento de la vitamina $\mathrm{D}$, o que identifiquen una relación de doble vía entre la enfermedad y la hipovitaminosis. Conclusión: Se concluyó que existe una fuerte asociación entre la hipovitaminosis D y un síndrome de inmunodeficiencia adquirida (SIDA), generalmente potenciado o condicionado por factores de riesgo intrínsecos o estilo de vida. Además, hubo una asociación entre la deficiencia de vitaminas en pacientes y una mayor vulnerabilidad a enfermedades oportunistas y, por el contrario, una reducción de infecciones, enfermedades crónicas y mortalidad en pacientes con VIH/SIDA con estadísticas de enfermedad D. Este fue el objetivo del estudio, también se identificaron correlaciones asociadas entre la disminución en el número de linfocitos T CD4. A pesar de esto, se enfatiza la necesidad de llevar a cabo más investigaciones que permitan una mayor aclaración sobre una propuesta temática.

Palabras clave: Vitamina D; Deficiencia; VIH/SIDA.

\section{Introdução}

A epidemia mundial de Síndrome da Imunodeficiência Adquirida (AIDS) ainda constitui-se um relevante problema de saúde pública, que acomete cerca de 36,7 milhões de pessoas no mundo, embora sejam inúmeros os avanços alcançados nos últimos anos (Levy, 2012; WHO, 2015). Segundo dados do Ministério da Saúde, desde 1980, até junho de 2018 foram computados 982.129 casos de pessoas infectadas pelo vírus da imunodeficiência humana (HIV) no Brasil, o que expressa uma importante causa de morbidade e mortalidade (Brasil, 2018). 
Dentre os vários mecanismos envolvidos no processo infeccioso ocasionado pelo vírus do HIV, destaca-se a ativação crônica do sistema imunitário, que provoca disfunção da homeostasia das células $\mathrm{T}$, indução do estresse oxidativo e depleção das concentrações dos antioxidantes citoplasmáticos (Ivanov, et al., 2016; Nkengfack, et al., 2012; Tonel, et al., 2018). Esses eventos, direta ou indiretamente, ocasionam aos indivíduos portadores do vírus, maiores chances de infecções oportunistas, haja vista o comprometimento do sistema imunológico.

Vale ressaltar que com o advento da terapia antirretroviral (TARV), a morbimortalidade reduziu consideravelmente em portadores de HIV, diminuindo a progressão da doença, devido o controle da multiplicação viral, além do aumento no intervalo de tempo para o surgimento da AIDS (Silva, 2015). Entretanto, ainda existem complicações desencadeadas pelo uso da TARV, comumente interações medicamentosas, que podem levar a lipodistrofia e a deficiências de micronutrientes da dieta (Leão, 2012; Wang, et al., 2017).

A nutrição nesse contexto, evidencia o papel fundamental que a alimentação detém para a saúde desse público, sobretudo por contribuir para a melhora na qualidade de vida e na resposta ao tratamento, bem como, pela redução da imunodeficiência e morbidade decorrentes de infecções. Em contrapartida, na ausência do consumo de qualidade, algumas condições clínicas podem ser identificadas, a exemplo da linfopenia, causa significativa da perda da capacidade de defesa do hospedeiro, e comumente observada em casos de deficiência de micronutrientes, com destaque para a vitamina D (Brasil, 2011).

Estudos recentes têm voltado a atenção para este nutriente por constatarem efeitos relevantes no cérebro, próstata, mama, cólon, coração, células do sistema imunológico, pâncreas e sistema cardiovascular (Dazini, et al., 2017; Ferraz, et al., 2018), além de evidenciar o seu desempenho satisfatório sobre o metabolismo do cálcio e ósseo, e outros eventos característicos de pacientes infectados pelo HIV (Hamill, et al., 2017).

No sistema imune, por exemplo, a vitamina D é potencialmente capaz de aumentar a expressão do gene da catelicidina (LL-37) nos macrófagos, que participam da atividade antibacteriana; e na imunidade adquirida, o micronutriente atua, principalmente, nas células $\mathrm{T}$, suprimindo a proliferação de células Th1, e a produção do interferon gama e da IL-2. Alguns achados já demonstram a capacidade do LL-37 de inibir a replicação do HIV-1 em linfócitos T CD+4 (CD4+LT) e macrófagos, corroborando para melhora do quadro dos portadores (Borges, et al., 2011; Musarurwa, et al., 2018; Schtscherbyna, et al., 2016). 
Com isso, sugere-se que concentrações de vitamina $\mathrm{D}$ abaixo do ideal podem afetar a resposta do sistema imunológico em pacientes com HIV/AIDS, como também, contribuir para o desenvolvimento de doenças crônicas e piora do prognóstico, considerando ainda a insuficiência de pontos de corte ideais do nutriente em questão, nesse grupo de adoecidos. Portanto, diante do exposto, este estudo objetivou investigar na literatura o conhecimento atual sobre a associação entre a deficiência de vitamina D e a Síndrome da Imunodeficiência Adquirida.

\section{Metodologia}

A pesquisa dos artigos foi realizada nas bases de dados SciELO, LILACS e PubMed, no mês de novembro de 2019, por duas autoras (BBMS e ELCO) de forma independente. As pesquisas realizadas pelas referidas autoras foram comparadas e discutidas sob o parecer de um terceiro revisor (TRN), pelo qual foi verificada a equivalência na busca e seleção de artigos.

A estratégia PICO foi utilizada para definição da pergunta norteadora dessa revisão, "Qual a associação entre baixas concentrações de vitamina D e Síndrome de Imunodeficiência Adquirida?". As palavras-chave (vitamina D, AIDS, HIV, imunidade) foram utilizadas no levantamento bibliográfico. Os seguintes descritores foram cruzados na busca dos artigos "vitamina D and AIDS", "status vitamina D and HIV", "vitamin D status and HIV", "Vitamina D and imunidade and HIV".

Foram incluídos nesta revisão integrativa ensaios clínicos, estudos originais, transversais, coorte e caso-controle, realizados em seres humanos, publicados em português, espanhol e inglês, sem limite de ano de publicação, e que associaram níveis baixos de vitamina D em pacientes com HIV/AIDS e suas implicações clínicas.

A seleção dos estudos ocorreu em três etapas. Inicialmente, por meio da análise dos títulos dos artigos, o examinador avaliou aqueles identificados pelas combinações dos descritores nas bases de dados propostas. Em seguida, após seleção dos títulos relevantes, foi realizada a leitura dos resumos. Nos casos em que existia a necessidade de obter maiores informações, o texto era explorado na íntegra para posteriormente ser julgado pertinente ao tema de estudo e, em seguida, analisado. Não foram incluídos nesta revisão relatos de caso, artigos de revisão e trabalhos indisponíveis na íntegra. Os detalhes da seleção são mostrados na Figura 1. 
Os aspectos avaliados foram: ano de publicação, idioma, desenho e duração do estudo, tamanho da amostra analisada, perdas, objetivo do estudo, principais resultados, estatística empregada. Para facilitar a análise e discussão, os dados foram organizados em tabelas.

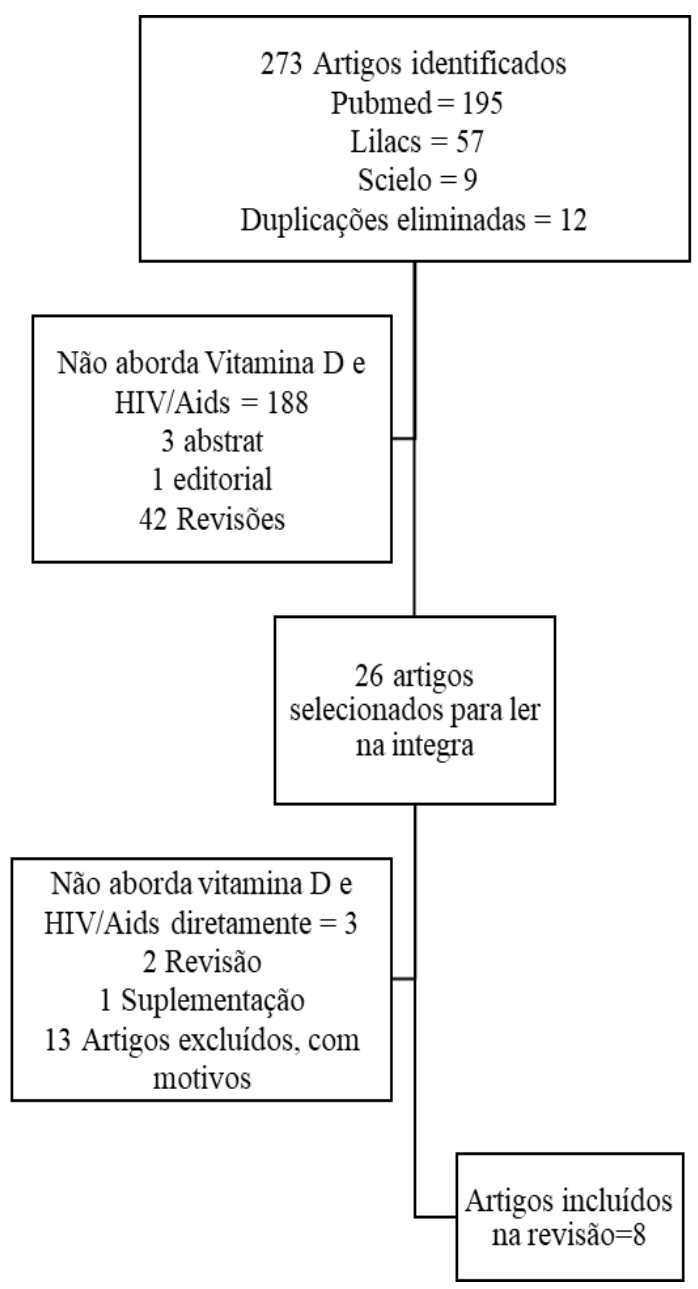

Figura 1. Fluxograma da seleção dos artigos. Teresina, 2019. 


\section{Resultados}

O levantamento bibliográfico recuperou 273 artigos, dos quais 12 eram duplicados, 188 não abordavam HIV, ou tangenciavam completamente a temática do estudo, totalizando 8 artigos que compuseram o corpus de análise da presente revisão. Os estudos selecionados caracterizam-se como originais do tipo coorte (3), transversal (3), observacional (1), randomizado duplo-cego (1), e em todos buscou-se avaliar o status de vitamina D em pacientes com HIV/AIDS. No Quadro 1 estão sintetizadas as principais informações dos estudos selecionados que incluem: Autor e ano, idioma da publicação e país de estudo; objetivos; metodologia aplicada (tipo de estudo, amostra, uso ou não de TARV); resultados.

Quadro 1. Estudos clínicos sobre o status de vitamina D e sua relação com HIV/AIDS. Teresina, 2019.

\begin{tabular}{|c|c|c|c|c|}
\hline Autor/Ano/País & Objetivo & $\begin{array}{l}\text { Tipo de } \\
\text { estudo }\end{array}$ & Caracterização & Principais resultados \\
\hline $\begin{array}{l}\text { Dúran, et al., } \\
\text { 2019. Espanhol. } \\
\text { [Argentina] }\end{array}$ & $\begin{array}{l}\text { Determinar a prevalência de } \\
\text { hipovitaminose D em um } \\
\text { estudo coorte em adultos } \\
\text { com HIV na cidade de } \\
\text { Buenos Aires. }\end{array}$ & Coorte & $\begin{array}{l}\mathrm{N}=814 ; \text { Idade }>18 \text { anos; } \\
\text { Excluídos: } \\
\text { suplementados com vit. D ou } \\
\text { com doença associada ao HIV. } \\
\text { Variáveis: vitamina D, } \\
\text { linfócitos, idade, sexo e } \\
\text { TARV. Ponto de corte de } \\
\text { vitamina D <20ng/dL. }\end{array}$ & $\begin{array}{l}99,9 \% \text { recebia tratamento TARV e } 22,5 \% \\
\text { apresentavam redução do número de linfócitos } \mathrm{T} \\
\mathrm{CD} 4 \text {. A prevalência de hipovitaminose } \mathrm{D} \text { foi de } \\
79,7 \% \text {. Não foi encontrado relação entre a } \\
\text { hipovitaminose e o tratamento ou o número de } \\
\text { linfócitos T CD4. }\end{array}$ \\
\hline $\begin{array}{l}\text { Musarurwa, et al., } \\
\text { 2018. Inglês. }\end{array}$ & $\begin{array}{l}\text { Examinar a prevalência de } \\
\text { deficiência de vitamina } \mathrm{D} \text { e }\end{array}$ & Coorte & $\begin{array}{l}\mathrm{N}=\quad 284^{*} \text { com } \quad \text { HIV } \\
\text { hospitalizados. }\end{array}$ & $\begin{array}{l}\text { A vitamina D apresentou-se com concentração } \\
\text { significativamente maior }(25.3 \mathrm{ng} / \mathrm{ml}) \text { no grupo }\end{array}$ \\
\hline
\end{tabular}




\begin{tabular}{|c|c|c|c|c|}
\hline [África] & $\begin{array}{l}\text { risco de tuberculose entre } \\
\text { pacientes hospitalizados } \\
\text { infectados pelo HIV com e } \\
\text { sem tuberculose. }\end{array}$ & & $\begin{array}{l}* 145 \text { com HIV + tuberculose e } \\
139 \text { sem tuberculose. Exames } \\
\text { laboratoriais (Linfócitos T } \\
\text { CD4+, hemograma e VitD). }\end{array}$ & $\begin{array}{l}\text { com tuberculose quando comparado com os } \\
\text { pacientes que possuíam apenas HIV } \\
(20,4 \mathrm{ng} / \mathrm{mL}) \text {, contudo as duas faixas apresentam- } \\
\text { se abaixo do recomendado. }\end{array}$ \\
\hline $\begin{array}{l}\text { Schtscherbyna, et } \\
\text { al., 2016. Inglês. } \\
\text { [Brasil] }\end{array}$ & $\begin{array}{l}\text { Determinar a prevalência e } \\
\text { os fatores relacionados à } \\
\text { insuficiência de vitamina D } \\
\text { (VitD) em adolescentes e } \\
\text { adultos jovens com o vírus } \\
\text { da imunodeficiência } \\
\text { humanarairida } \\
\text { perinatalmente adquila }\end{array}$ & Coorte & $\begin{array}{l}\text { N=65 pacientes; Variáveis: } \\
\text { nutrição, paratormônio sérico e } \\
\text { 25-hidroxivitamina D sérica } \\
\text { [s25 (OH)D](classificada em } \\
\text { insuficiência quando }<30 \\
\text { ng/mL). }\end{array}$ & $\begin{array}{l}\text { A média de s25 }(\mathrm{OH}) \mathrm{D} \text { foi de } 37,7 \text { e } 29,2 \% \\
\text { apresentaram insuficiência de VitD. Não houve } \\
\text { diferença entre o status VitD e classificação } \\
\text { clínica, imunológica, tipo de TARV. Somente o } \\
\text { consumo de VitD apresentou tendência de } \\
\text { associação com s25 (OH). Os indivíduos } \\
\text { analisados no verão/outono apresentaram s25 } \\
(\mathrm{OH}) \mathrm{D} \text { superior aos analisados no } \\
\text { inverno/primavera. }\end{array}$ \\
\hline $\begin{array}{l}\text { Canuto, et al., } \\
\text { 2015. Português. } \\
\text { [Brasil] }\end{array}$ & $\begin{array}{l}\text { Investigar fatores de risco } \\
\text { associados } \\
\text { hipovitaminose } \quad \mathrm{D} \text { em } \\
\text { pacientes adultos infectados } \\
\text { por HIV/AIDS, num centro } \\
\text { de referência em Maceió- } \\
\mathrm{AL}\end{array}$ & Transversal & $\begin{array}{l}\mathrm{N}=125 ; \quad \text { Variáveis: exame } \\
\text { físico, concentrações de } \\
\text { vitamina } \mathrm{D} \text {. }\end{array}$ & $\begin{array}{l}\text { Observou-se níveis de vitamina } D \text { mais altos nas } \\
\text { mulheres, em pacientes que não faziam uso de } \\
\text { filtro solar e naqueles com infecções } \\
\text { oportunistas pregressas. Valores mais baixos } \\
\text { foram associados ao uso de antirretrovirais, } \\
\text { sobrepeso e obesidade. }\end{array}$ \\
\hline
\end{tabular}




\begin{tabular}{|c|c|c|c|c|}
\hline $\begin{array}{l}\text { Schwartz, et al., } \\
\text { 2014. Inglês. } \\
\text { [EUA] }\end{array}$ & $\begin{array}{l}\text { Avaliar a relação da } \\
\text { vitamina D com o estado do } \\
\text { HIV, o tratamento do HIV, } \\
\text { em uma coorte de } \\
\text { infectados pelo HIV de } \\
\text { meia-idade e mulheres não } \\
\text { infectadas inscritas no } \\
\text { estudo. }\end{array}$ & $\begin{array}{l}\text { Transversal, } \\
\text { com } \\
\text { subgrupos } \\
\text { controles }\end{array}$ & $\begin{array}{l}\text { N=1.758* participantes. } * 507 \\
\text { não soropositivo, } 358 \text { com HIV } \\
\text { + sem TARV e } 893 \text { com HIV + } \\
\text { TARV. }\end{array}$ & $\begin{array}{l}\text { Dentre os participantes, } 63 \% \text { possuíam } \\
\text { deficiência na vitamina D. O grupo com HIV } \\
\text { não tratado possui mais deficiência na vitamina } \\
\text { D, quando comparado aos demais grupos. }\end{array}$ \\
\hline $\begin{array}{l}\text { Eckard, et al., } \\
\text { 2013. Inglês. } \\
\text { [EUA] }\end{array}$ & $\begin{array}{l}\text { Determinar o status de } \\
\text { vitamina D em gestantes } \\
\text { infectadas pelo HIV nos } \\
\text { Estados Unidos e investigar } \\
\text { associações com a } \\
\text { deficiência de vitamina D. }\end{array}$ & $\begin{array}{l}\text { Transversal } \\
\text { multicêntrico, } \\
\text { do tipo caso- } \\
\text { controle }\end{array}$ & $\begin{array}{l}\mathrm{N}=40 \quad \text { mulheres grávidas } \\
(\mathrm{IG}>20 \text { semanas); } 16 \text { com } \\
\text { HIV+ (em uso de TARV) e } 24 \\
\text { saudáveis. Excluídos: mulher } \\
\text { com doença crônica associada. } \\
\text { Adotou-se o valor de referência } \\
\text { de } 20 \mathrm{ng} / \mathrm{dL} \text { de vitamina D } \\
\text { como ponto de corte. }\end{array}$ & $\begin{array}{l}\text { Mulheres com HIV tinham deficiência de } \\
\text { vitamina D }(70 \%) \text { e } 25 \% \text { eram insuficientes. Das } \\
\text { mulheres infectadas } 56 \% \text { estavam na faixa } \\
\text { severamente deficiente. Contudo, a deficiência } \\
\text { de vitamina D também foi observada no grupo } \\
\text { controle, } 67 \% \text { dos indivíduos. Desse modo não } \\
\text { houve diferença significativa entre mulheres com } \\
\text { HIV e o grupo controle. }\end{array}$ \\
\hline $\begin{array}{l}\text { Adeyemi, et al., } \\
\text { 2011. Inglês. } \\
\text { [EUA] }\end{array}$ & $\begin{array}{l}\text { Determinar a prevalência e } \\
\text { preditores de deficiência de } \\
\text { vitamina D em pessoas de } \\
\text { meia idade e etnicamente } \\
\text { diversas infectadas pelo } \\
\text { HIV e mulheres não } \\
\text { infectadas inscritas no } \\
\text { estudo Interagency } \\
\text { Women's HIV (WIHS). }\end{array}$ & $\begin{array}{l}\text { Coorte, } \\
\text { prospectivo, } \\
\text { multicêntrico. }\end{array}$ & $\begin{array}{l}\mathrm{N}=1778 \text { mulheres de seis } \\
\text { cidades, } 1268 \text { soropositivas e } \\
510 \text { não soropositivas. } \\
\text { Variáveis=exames } \\
\text { exames físicos; } \\
\text { ginecológico. }\end{array}$ & $\begin{array}{l}58 \% \text { das mulheres HIV+ eram afro- } \\
\text { descendentes, e } 24 \% \text { caucasianas, com idade } \\
\text { média de } 44 \text { anos. A deficiência de vitamina D } \\
\text { foi presente nos dois grupos, com } 60 \% \text { e } 72 \% \text {, } \\
\text { para o grupo HIV+ e para o HIV-, } \\
\text { respectivamente. Foram observados que a } \\
\text { deficiência em vitamina D aumenta com a } \\
\text { presença de fatores como o fumo, excessso de } \\
\text { peso e a etnia. }\end{array}$ \\
\hline \multicolumn{5}{|c|}{9} \\
\hline
\end{tabular}


Research, Society and Development, v. 9, n. 4, e85942679, 2020

(CC BY 4.0) | ISSN 2525-3409 | DOI: http://dx.doi.org/10.33448/rsd-v9i4.2679

\begin{tabular}{|c|c|c|c|c|}
\hline $\begin{array}{l}\text { Conrado, et al., } \\
\text { 2010. Português. } \\
\text { [Brasil] }\end{array}$ & $\begin{array}{l}\text { Investigar a possível relação } \\
\text { existente entre a deficiência } \\
\text { de vitamina D e o aumento } \\
\text { de perda óssea e doença } \\
\text { cardiovascular } \quad \text { em } \\
\text { indivíduos com HIV. }\end{array}$ & Observacional & Não informado & $\begin{array}{l}\text { Alta frequência de hipertensão, tabagismo, } \\
\text { dislipidemia e diabetes; risco de infarto do } \\
\text { miocárdio. Expostos a outros fatores de risco, } \\
\text { incluindo a própria doença, o efeito do vírus nos } \\
\text { vasos sanguíneos, inflamação e complicações } \\
\text { por uso prolongado da terapia antirretroviral. }\end{array}$ \\
\hline $\begin{array}{l}\text { Mehta, et al., } \\
\text { 2010. Inglês. } \\
\text { [África] }\end{array}$ & $\begin{array}{l}\text { Avaliar o status de vitamina } \\
\text { D com relevância para a } \\
\text { infecção pelo HIV. }\end{array}$ & $\begin{array}{l}\text { Randomizado } \\
\text { duplo-cego }\end{array}$ & $\begin{array}{l}\text { N=884* mulheres grávidas } \\
\text { HIV+. Variáveis: laboratoriais } \\
\text { (fezes, urina, sangue), } \\
\text { ginecológico; } r \text { medidas } \\
\text { antropométricas, concentrações } \\
\text { de vitamina D, linfócitos T } \\
\text { CD4+, CD8, CD3. Tempo } \\
\text { médio de acompanhamento foi } \\
\text { de } 69,5 \text { meses. *Realocadas } \\
\text { em subgrupos, sem uso da } \\
\text { TARV. }\end{array}$ & $\begin{array}{l}\text { Da amostra } 39,3 \% \text { apresentou baixa } \\
\text { concentração de vitD, e entre } 83,1 \% \text { que estavam } \\
\text { em estágio I, } 37,8 \% \text { tinha deficiência de vitD. } \\
\text { Não houve diferença significativa na contagem } \\
\text { de células T CD4 entre as mulheres, } \\
\text { independente da concentração de vitD. Durante o } \\
\text { estudo, } 32,5 \% \text { das mulheres morreram, destas } \\
70 \% \text { por causa da AIDS. } 60,3 \% \text { das mulheres } \\
\text { com estágio I e II evoluíram para o estágio III ou } \\
\text { superior, sendo } 1,25 \text { vezes maior em mulheres } \\
\text { com deficiência de vitamina D. }\end{array}$ \\
\hline
\end{tabular}

TARV: Terapia antirretroviral; HIV: Vírus da imunodeficiência humana; HIV+: Soropositivo; HIV/TB+: Vírus da imunodeficiência humana/tuberculose (co-infecção); OMS:

Organização Mundial da Saúde. FONTE: Dados da Pesquisa. Teresina, 2019. 


\section{Discussão}

Apesar das publicações apresentarem grupos distintos, foi perceptível que a deficiência de vitamina D e a AIDS estiveram intimamente correlacionadas. O estudo de Canuto, et al., (2015), por exemplo, observou que a prevalência de deficiência de vitamina D em pacientes infectados pelo HIV alcançou taxas que variaram de 10 a $73 \%$ e estiveram significativamente associados com tradicionais fatores de risco para hipovitaminose $\mathrm{D}$, como idade mais avançada, sexo feminino, baixa exposição ao sol, pele escura, doenças genéticas do receptor de vitamina $\mathrm{D}$, maior índice de massa corporal, distúrbios de absorção gastrointestinal e no fígado, ou doença renal (Hollick, et al., 2009; Oydele, et al., 2012).

As baixas concentrações de vitamina D têm sido observadas em $41 \%$ a $90 \%$ dos pacientes infectados pelo HIV (Conrado, et al., 2010; Rodriguez, et al., 2009). Os estudos de Adeyemi, et al., (2011) e Schwartz, et al., (2014), demostraram que mais de 60\% dos participantes da pesquisa apresentavam deficiência de vitamina $D$, com fatores de risco tradicionais dessa carência, destacando-se a idade avançada, o tabagismo, etnia afroamericana ou hispânica e IMC elevado.

Vale ressaltar que a principal fonte de $25(\mathrm{OH})$ D é a exposição a luz solar, por meio da qual são fornecidos níveis séricos adequados desse hormônio para a maioria dos brasileiros (Rutstein, et al., 2011), embora tenha sido constatado que essa exposição ainda é inadequada na maioria dos estudos realizados em países tropicais como África, Brasil, Argentina, justificada pela alarmante prevalência de hipovitaminose D.

Analisando tal fato, o estudo de Schtscherbyna, et al., (2016), realizado com adolescentes e adultos jovens com infecção pelo vírus do HIV, perinatalmente adquirida, e que foi desenvolvido em um estado ensolarado, observou que os participantes da pesquisa apresentaram prevalência moderada de insuficiência de Vitamina D, sugerindo que a exposição à luz solar reduz o número de casos graves de hipovitaminose D.

Considerando especificamente esses casos, o estudo de Conrado, et al., (2010) constatou que tal deficiência tem sido observada como possível condição de risco para eventos metabólicos e cardiovasculares, e também para o aumento da prevalência da AIDS, o que aponta para a necessidade de estabelecer medidas de prevenção. Os autores citam ainda que a suplementação de vitamina $\mathrm{D}$ torna-se necessária, tendo exposição ao sol como complementar. 
Paralelamente, a hipovitaminose $\mathrm{D}$ foi relacionada ao surgimento de doenças oportunistas como a tuberculose pulmonar, que segundo estudo de Musarurwa, et al., (2018) demonstrou que pacientes HIV+ (soropositivos), e HIV+/TP+ (soropositivos e com tuberculose pulmonar) apresentaram deficiência de vitamina $\mathrm{D}$, e que essa carência pode estar vinculada também ao aumento da incidência de osteoporose durante o tratamento crônico do HIV (Brown \& Mccomsey, 2010; Hsue, et al., 2009).

Nos estudos de Dúran, et al., (2019) e Eckard, et al., (2013) a prevalência de deficiência de vitamina $\mathrm{D}$ esteve presente em todos os grupos de avaliados (controle ou soropositivos), não existindo correlações significativas entre a concentração de vitamina D e o quantitativo de linfócitos T CD4, embora constatada a diminuição destes, no grupo de soropositivos. Semelhantemente, Mehta, et al., (2010), não identificou associação significativa entre os valores de vitamina $\mathrm{D}$, número de células $\mathrm{T}$ e mortalidade por AIDS.

No estudo de Mehta, et al., (2010) não houve relação entre mortalidade por causas relacionadas e AIDS, tampouco entre mortalidade por causas gerais e concentrações de vitamina D. Em contrapartida, mulheres com valores elevados do nutriente, apresentaram um risco de mortalidade $42 \%$ menor por causas gerais, se comparado a mulheres com concentrações deficientes da vitamina. Em análises com níveis contínuos, comprovou-se que o risco de mortalidade é diretamente proporcional às concentrações de vitamina $\mathrm{D}$.

Em se tratando da utilização de terapia antirretroviral, os estudos de Musarurwa, et al., (2018) e Schwartz, et al., (2014) demonstraram que pacientes que faziam TARV apresentavam maiores concentrações de vitamina $\mathrm{D}$, sem diferença significativa em relação aos que não faziam uso. Esse achado contrasta com literaturas que afirmam que a TARV ocasiona a diminuição na concentração de vitamina D.

No que se refere a deficiências associadas, foi constatada uma forte significância entre níveis de vitamina D, anemia e marcadores de deficiência de ferro. Segundo Mehta, et al., (2010), mulheres com baixas concentrações da vitamina, apresentaram um risco $46 \%$ maior de desenvolver anemia grave durante o acompanhamento em modelos multivariados, o que não foi constatado na condição inversa. Ainda observaram que as baixas concentrações foram significativamente associadas ao aumento do risco de progressão da doença do HIV, anemia grave e microcitose hipocrômica em mulheres tanzanianas infectadas pelo HIV.

A associação protetora da vitamina D frente à progressão da doença do HIV pode ser explicada por seu papel na função imunológica, uma vez que promove a melhora funcional dos macrófagos, da imunidade mediada por células, bem como, aumenta o número de natural 
killers e a atividade citolítica, sugerindo um papel importante na prevenção de infecções. Estudos laboratoriais identificaram vários mecanismos pelos quais a vitamina $\mathrm{D}$ poderia retardar a progressão da doença do HIV (Villamor, 2006), por exemplo, pela forma 1,25 $(\mathrm{OH}) 2 \mathrm{D}$, que diminui a expressão do receptor de superfície CD4 na linha de células de leucemia promielocítica e monócitos humanos.

No que tange a relação da vitamina $\mathrm{D}$ com receptores de células imunológicas protetoras constata-se que receptores semelhantes ao Toll Like (TLRs) (Liu, et al., 2006), desempenham na imunidade inata, a função seletiva quanto a entrada de patógenos, pelo estímulo à produção da forma ativa da vitamina $\mathrm{D}$, sendo esta capaz de induzir a atividade antibacteriana. Este mecanismo tem se mostrado importante na resposta do sistema imunológico e proteção a outras doenças oportunistas, exemplificada pela tuberculose.

Acredita-se que pela ativação do TLR de macrófagos humanos reguladores da expressão de VDRs, (vitamin D receptors) a enzima (CYP27b1) catalisa a conversão de 25 $(\mathrm{OH})$ D para 1,25 $(\mathrm{OH}) 2 \mathrm{D}$, originando o metabólito biologicamente ativo de vitamina $\mathrm{D}$. Logo, na presença de concentrações adequadas de $25(\mathrm{OH}) \mathrm{D}$, a autorregularão de vitamina D reativa leva à indução de peptídeos antimicrobianos com ação direta contra patógenos intracelulares, incluindo Mycobacterium tuberculosis (Liu, et al., 2006), uma das principais causas de progressão e mortalidade da doença entre os pacientes infectados pelo HIV. Para esses casos, o aumento da resistência à tuberculose pode potencialmente prolongar a sobrevida nesses pacientes (Mehta, et al., 2010).

\section{Conclusão}

Concluiu-se que a maioria dos estudos desta revisão apontou para a existência de associação entre hipovitaminose D e a síndrome da imunodeficiência adquirida (AIDS) geralmente potencializada ou condicionada a fatores de risco como excesso de peso, localização geográfica, idade avançada, etnia, baixa exposição ao sol e hábito de fumar.

Além disso, os resultados sugerem também uma possível relação inversa de proporcionalidade entre concentrações da vitamina em portadores e risco para doenças oportunistas, embora alguns achados demonstrem que pacientes com HIV e coinfeccionados, podem apresentar concentrações de vitamina D aumentadas, se comparados ao grupo HIV+, sem infecções associadas. Esse dado conflitante possivelmente pode ser explicado por 
desregulações nos mecanismos metabólicos, inflamatórios e celulares, e patofisiológicos característicos.

Foram identificadas também correlações significativas entre a diminuição do número de células linfócitos T CD4 e os níveis de vitamina D, pontuando a necessidade de mais estudos para fundamentação. E por fim, verificou-se ainda associação entre concentrações adequadas de vitamina $\mathrm{D}$ e redução de infecções, de doenças crônicas e da mortalidade em portadores de HIV/AIDS.

Contudo, não se exime a necessidade da realização de mais pesquisas que permitam configurar um perfil mais claro e de maior acurácia sobre a relação do HIV com a hipovitaminose D, com vistas a elucidar aspectos pouco discutidos, a saber: o grau de influência da ativação da vitamina $\mathrm{D}$, a relação da TARV com quantitativo de vitamina $\mathrm{D}$, e linfócitos T nesse grupo específico.

\section{Referências}

Adeyemi, O. M., Agniel, D., French, A. L., Tien, P., Weber, K., Glesby, M. J., \& Meyer, W. (2011). Vitamin D deficiency in HIV-infected and un-infected women in the US. Journal of acquired immune deficiency syndromes (1999), 57(3), 197.

Borges, M. C., Martini, L. A., \& Rogero, M. M. (2011). Current perspectives on vitamin D, immune system, and chronic diseases. Nutrition, 27(4), 399-404.

Brasil (2011). Projeto Diretrizes. Terapia Nutricional na Síndrome da Imunodeficiência Adquirida (HIV/AIDS). Sociedade Brasileira de Nutrição Parenteral e Enteral \& Associação Brasileira de Nutrologia.

Brasil (2018). Ministério da Saúde. Secretaria de Vigilância em Saúde. Departamento de DST, AIDS e Hepatites Virais. Boletim Epidemiológico-AIDS/HIV. Brasília: Ministério da Saúde.

Brown, T. T., \& McComsey, G. A. (2010). Short communications-association between initiation of antiretroviral therapy with efavirenz and decreases in 25-hydroxyvitamin D.Antiviral therapy, 15, 425429.

Canuto, J. M. P., Canuto, V. M. P., Lima, M. H. A. D., Omena, A. L. C. S. D., Morais, T. M. D. L., Paiva, A. M., \& Ferreira, S. M. S. (2015). Risk factors associated with hypovitaminosis D in HIV/AIDS-infected adults. Archives of endocrinology and metabolism, 59(1), 34-41.

Conrado, T., Miranda-Filho, D. D. B., \& Bandeira, F. (2010). Deficiência de vitamina D em indivíduos infectados pelo HIV: mais um fator de risco para perda óssea e doença cardiovascular? Arq Bras Endocrinol Metab, 54(2), 118-122.

Dazini, PO., Lanna, CMM., \& Moreira, APB (2017). Relação entre vitamina D e cálcio no desenvolvimento do Diabetes Mellitus tipo 1 e 2-Uma revisão de literatura. HU Revista, 43 (2), 163172. 
Vanesa Durán, M. A. R. Í. A., Sabato, S., Sánchez Thomas, D. I. E. G. O., Greco, D., \& Lopardo, G. (2019). Alta prevalencia de hipovitaminosis d en personas infectadas con hiv atendidas en un centro ambulatorio de la ciudad de Buenos Aires. Medicina (Buenos Aires), 79 (5).

Eckard, A. R., Tangpricha, V., Seydafkan, S., O’Riordan, M. A., Storer, N., Labbato, D., \& McComsey, G. A. (2013). The relationship between vitamin D status and HIV-related complications in HIV-infected children and young adults. The Pediatric infectious disease journal, 32(11), 1224.

Hsue, P. Y., Hunt, P. W., Schnell, A., Kalapus, S. C., Hoh, R., Ganz, P., ... \& Deeks, S. G. (2009). Role of viral replication, antiretroviral therapy, and immunodeficiency in HIV-associated atherosclerosis. AIDS (London, England), 23(9), 1059.

Ferraz, C. L. H., Mendes, A. D. N., Ferraz, T. M. B. L., \& da Silva, C. A. B. (2018). Associação entre vitamina $\mathrm{D}$, controle glicêmico e complicações microvasculares no diabetes tipo 1. Revista Brasileira em Promoção da Saúde, 32 (2).

Guimarães, M. D. C., Carneiro, M., Abreu, D. M. X. D., \& França, E. B. (2017). Mortalidade por HIV/AIDS no Brasil, 2000-2015: motivos para preocupação? Revista Brasileira de Epidemiologia, 20 (1), 182-190.

Hamill, M. M., Pettifor, J. M., Ward, K. A., Norris, S. A., \& Prentice, A. (2017). Changes in Bone Mineral Density, Body Composition, Vitamin D Status, and Mineral Metabolism in Urban HIV-Positive South African Women Over 12 Months. Journal of Bone and Mineral Research, 32 (8), 1615-1624.

Holick, M. F. (2009). Vitamin D: the other steroid hormone for muscle function and strength. Menopause, 16 (6), 1077-1078.

Ivanov, A. V., Valuev-Elliston, V. T., Ivanova, O. N., Kochetkov, S. N., Starodubova, E. S., Bartosch, B., \& Isaguliants, M. G. (2016). Oxidative stress during HIV infection: mechanisms and consequences. Oxidative medicine and cellular longevity, 2016, 1-18.

Khazai, N., Judd, S.E., \& Tangpricha, V., (2008). Calcium and vitamin D: skeletal and extraskeletal health. Current rheumatology reports, 10 (2), 110-117.

Leão, L, \& Gomes, M. (2012). Manual de Nutrição Clínica: Para atendimento Ambulatorial do Adulto. 12 ed. Petrópolis, RJ: Vozes.

Levy, J. A., Autran, B., Coutinho, R. A., \& Phair, J. P. (2012). 25 Years of AIDS: recording progress and future challenges. AIDS, 26, 1187-1189.

Liu, P. T., Stenger, S., Li, H., Wenzel, L., Tan, B. H., Krutzik, S. R., \& Kamen, D. L. (2006). Toll-like receptor triggering of a vitamin D-mediated human antimicrobial response. Science, 311, 1770-1773.

Mehta, S., Giovannucci, E., Mugusi, F. M., Spiegelman, D., Aboud, S., Hertzmark, E., \& Fawzi, W. W. (2010). Vitamin D status of HIV-infected women and its association with HIV disease progression, anemia, and mortality. PloS one, 5 (1), 8770.

Musarurwa, C., Zijenah, L. S., Mhandire, D. Z., Bandason, T., Mhandire, K., Chipiti, M. M., \& Mujaji, W. B. (2018). Higher serum 25-hydroxyvitamin D concentrations are associated with active pulmonary tuberculosis in hospitalised HIV infected patients in a low income tropical setting: a cross sectional study. BMC pulmonary medicine, 18 (1), 67. 
Nkengfack, G. N., Torimiro, J. N., \& Englert, H. (2012). Effects of antioxidants on CD4 and viral load in HIV-infected women in sub-Saharan Africa-Dietary supplements vs. local diet. Int. J. Vitam. Nutr. Res, 82 (1), 63-72.

Oyedele, T., \& Adeyemi, O. M. (2012). High prevalence of vitamin D deficiency in HIV-infected adults: what are the future research questions? Curr HIV/AIDS Rep, 9 (1), 1-4.

Pugliese, C. et al (2014). Avaliação do status de antioxidantes e atividade da superóxido dismutase em crianças infectadas pelo HIV. Braz J Infect Dis, 18 (5).

Rodriguez, M., Daniels, B., Gunawardene, S., \& Robbins, G. K. (2009). High frequency of vitamin D deficiency in ambulatory HIV-positive patients. AIDS research and human retroviruses, 25 (1), 9-14.

Rutstein, R., Downes, A., Zemel, B., Schall, J., \& Stallings, V. (2011). Status de vitamina D em crianças e adultos jovens com infecção por HIV adquirida perinatalmente. Clin Nutr, 30, 624-628.

Schtscherbyna, A., et al (2016). Esta do da vitamina D em uma coorte brasileira de adolescentes e adultos jovens com infecção pelo vírus da imunodeficiência humana adquirida perinatalmente.

Mem. Inst. Oswaldo Cruz, 111 (2).

Schwartz, J. B., Moore, K. L., Yin, M., Sharma, A., Merenstein, D., Islam, T., \& Adeyemi, O. M. (2014). Relationship of vitamin D, HIV, HIV treatment, and lipid levels in the Women's Interagency HIV Study of HIV-infected and uninfected women in the United States. Journal of the International Association of Providers of AIDS Care (JIAPAC), 13 (3), 250-259.

Silva, I.G., Santos, U.O., \& Ferreira, E.S., (2015). A evolução do HIV/AIDS na terceira idade: uma revisão bibliográfica. Biblioteca Virtual de Enfermagem.

Tonel, D., Silva, T. O., Lazarotto, A. K., Muller, D. A., Lucca, L. D., Ichikawa, T. T. D., \& Gallina, A. L. (2018). Avaliação do status oxidativo, consumo alimentar de zinco e zinco plasmático em indivíduos infectados pelo HIV. Revista Brasileira de Análises Clínicas. 50(2), 153-60.

Vannucchi, H., Marchini, J. S., (2007). Nutrição Clínica. Rio de Janeiro: Guanabara Koogan.

Villamor, E., (2006). A potential role for vitamin D on HIV infection?. Nutrition reviews, 64 (5), 226233.

Wang, H., Chen, W., Li, D., Yin, X., Zhang, X., Olsen, N., \& Zheng, S. G. (2017). Vitamin D and chronic diseases. Aging and disease, 8 (3), 346.

Who (2015). World Health Organization. Global summary of the HIV/AIDS epidemic, 2014. 2015.

\section{Porcentagem de contribuição de cada autor no manuscrito}

Beatriz Bianca Miranda Silva - 25\%

Érica Letícia Carvalho de Oliveira - 25\%

Thaís Rodrigues Nogueira - 25\%

Betânia de Jesus e Silva de Almendra Freitas - 25\% 